\title{
Mitigation of Adverse Effects Caused by Shock Wave Boundary Layer Interactions through Optimal Wall Shaping
}

\author{
May-Fun Liou*, and Byung Joon Lee** \\ Corresponding author: may-fun.liou@nasa.gov
}

\begin{abstract}
It is known that the adverse effects of shock wave boundary layer interactions in high speed inlets include reduced total pressure recovery and highly distorted flow at the aerodynamic interface plane (AIP). This paper presents a design method for flow control which creates perturbations in geometry. These perturbations are tailored to change the flow structures in order to minimize shock wave boundary layer interactions (SWBLI) inside supersonic inlets. Optimizing the shape of two dimensional micro-size bumps is shown to be a very effective flow control method for two-dimensional SWBLI. In investigating the three dimensional SWBLI, a square duct is employed as a baseline. To investigate the mechanism whereby the geometric elements of the baseline, i.e. the bottom wall, the sidewall and the corner, exert influence on the flow's aerodynamic characteristics, each element is studied and optimized separately. It is found that arrays of micro-size bumps on the bottom wall of the duct have little effect in improving total pressure recovery though they are useful in suppressing the incipient separation in three-dimensional problems. Shaping sidewall geometry is effective in redistributing flow on the side wall and results in a less distorted flow at the exit. Subsequently, a near 50\% reduction in distortion is achieved. A simple change in corner geometry resulted in a $2.4 \%$ improvement in total pressure recovery.
\end{abstract}

\section{Introduction}

The three-dimensional shock wave boundary layer interaction problem has been studied extensively in the past thirty years. Flow separation in supersonic inlet due to shock wave boundary layer interaction can significantly reduce the pressure recovery by creating vortices which introduce distortion and instability at the aerodynamic interface plane (AIP) ${ }^{1}$. Thus, effective flow control has been an important subject and has been utilized to provide the necessary mass, momentum and/or energy to prevent flow separation.

To reduce the separation induced by SWBLIs, flow control techniques have traditionally been classified as active or passive, depending on whether or not the control involves power consumption. One commonly used active flow control method is to bleed out some of the low momentum boundary layer flows through arrays of small bleed holes ${ }^{2,3,4}$, slots or scoops. In the passive flow control camp, one example is vortex generators ${ }^{5,6,7}$ which generate stream-wise vortices and, thus, increase mixing the low momentum air in the boundary layer and the higher momentum core flow. Both control methods are designed to energize the boundary layer to withstand adverse pressure gradients and thus reduce the likelihood of flow separation within supersonic inlets. But the bleed system, though effective, makes the inlet more mechanically complex and heavier while the vortex generator (e.g. micro-ramp), though able to suppress local separation, results in little overall performance improvement ${ }^{1}$. Recently, hybrid techniques employing passive and active flow control in tandem have gained success and interest ${ }^{1,8}$.

Combining flow control and optimization techniques to control fluid motion for maximum performance, or so called flow control and optimization, has been of great engineering interest experimentally and computationally $^{9,10}$. Early works on controlling fluid motions were done through conventional trial-and-error methods which have technical difficulties and have prevented flow control and optimization from being used in complicated flow field, for instance, the aircraft propulsion system. More recently computational flow control and optimization has become practical in finding the optimized shape or boundary because of effective modern state-of-the-art optimization methodologies and reliable high-fidelity flow analyses by using Computational Fluid Dynamics (CFD), with help from the fast development of computer and computing technologies. Thus, instead of using disruptive penalty-ridden flow control devices, the concept of flow control can also be achieved by creating tailored perturbations in body geometry in attempts to deliberately change the flow structures and tendencies. This concept has been demonstrated in optimization of boundary-layer-ingestion (BLI) subsonic 
inlets through the wall shaping by Lee et al. ${ }^{11}$ Their results indicate the optimized geometry of an inlet diffuser satisfying objectives of minimum flow distortion and maximum total pressure recovery at the engine fan face energizes the expansion-driven separated flow at the inlet entrance. As a result, improvements in distortion as well as total pressure recovery at on- and off-design conditions are obtained simultaneously. In another example, Shukla et al. ${ }^{12}$ used a RANS model and the gradient-based optimization method to redesign NASA P2 and P8 inlets resulting in a near elimination of the cowl shock. Similarly, the goal of present work is to use the same concept in seeking an optimized geometry such that it is shaped to control flow separation caused by SWBLIs in supersonic inlets and to maximize the total pressure recovery. To facilitate the proposed flow control method, a well-studied rectangular supersonic inlet featuring strong three-dimensional SWBLIs and separations is used as the baseline case in this study.

Within rectangular supersonic inlets, the oblique SWBLI can be classified into three types: incident shock impingement and reflection on the bottom wall, glancing sidewall shock wave interactions and corner shock wave interactions ${ }^{13}$. The sensitivity of the physical mechanisms which are responsible for the generation of these three types of interaction to the proposed flow control method is investigated by isolating the geometry perturbations on bottom floor, side wall and corner respectively and separately. Therefore, the effect resulted from optimizing the shape of each surface in a box-type inlet has on the characteristics of its corresponding boundary layer can be examined. These optimized solutions can provide insight to the supersonic inlet design.

For this study, because the design problem contains multiple competing objectives such as minimizing pressure loss, minimizing flow distortion and maximizing aerodynamic performance, a multi-objective optimizer must be used. The Non-dominating, Sorting Genetic Algorithm, NSGA-II ${ }^{14}$ is employed to design a supersonic inlet with maximized aerodynamic performance, and to provide optimal solutions with respect to selected design parameters.

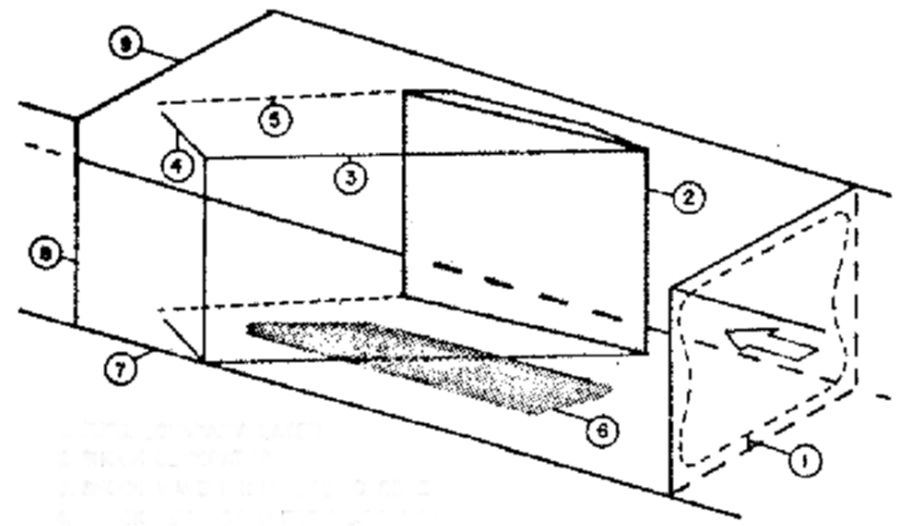

(a) Experimental test section ${ }^{16}$

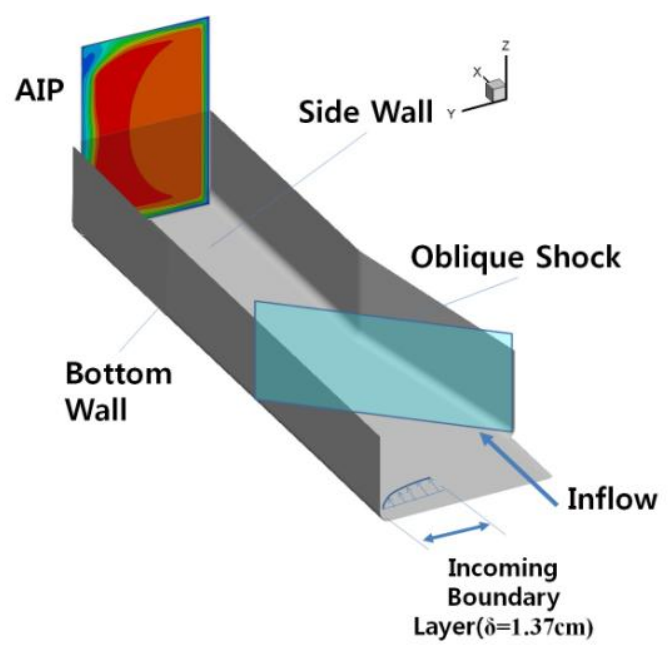

(b) Computational test section

Fig. 1: Schematic diagram of experimental and computational test sections. The schematic on the left is taken from Oskam et al. where (1) Turbulent Boundary Layer, (2) $10^{\circ}$ Shock generator, (3) Shock wave from leading edge, (4) Shock reflected from bottom, (5) Expansion from trailing edge, and (6) Region of experimental study on the side wall.

\section{CFD analysis}

A well validated three-dimensional Reynolds-averaged Navier-Stokes solver and a two-equation $k-w$-SST turbulence model are employed ${ }^{15}$. It is third-order accurate in space with a good selection of flux schemes. In the experimental setup of Oskam et al. ${ }^{16}$, shown in Fig. 1, a $10^{\circ}$ wedge was mounted off the wind tunnel wall to avoid the wall boundary layer at the leading edge of the wedge. The incoming boundary layer thickness of the bottom flat plate $(\mathrm{Y}=20 \mathrm{~cm})$ was about $1.37 \mathrm{~cm}$ at the entrance plane. The cross-section of the wind tunnel is 20 
$\mathrm{cm}$ by $20 \mathrm{~cm}$. Test conditions are: $M_{0}=2.94, T_{0}=290.0 \mathrm{~K}$, and free stream Re $=6.75 \times 10^{7} / \mathrm{m}$. The data measured includes yaw angles, static pressures and pitot pressure profiles. The details of the experimental rake locations are given in Ref. 16.

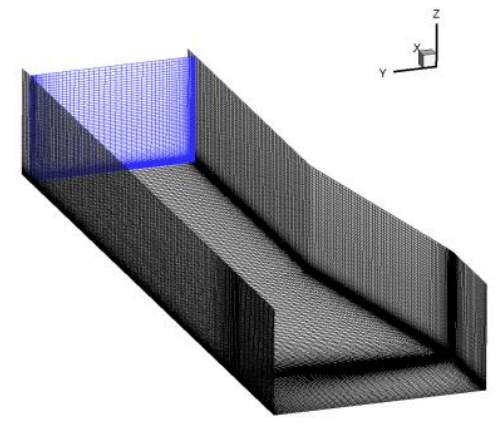

(a) Grid system (Coarse grid: 298x81x65)

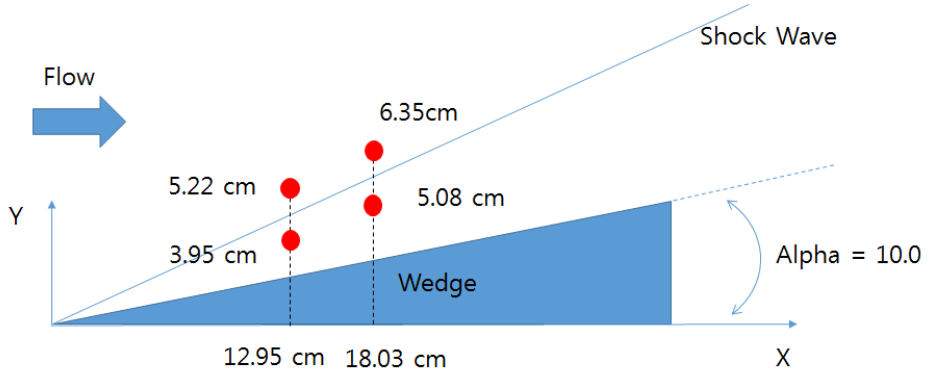

(b) Positions of experimental survey planes

Fig. 2: Grid system and positions of experimental survey planes in Oskam et al.

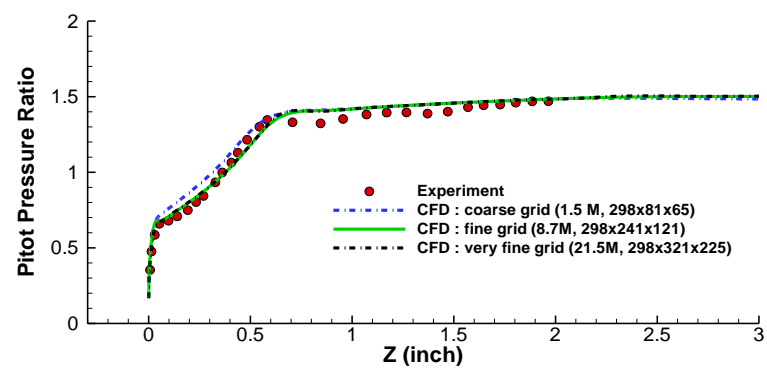

(a) $y=3.95 \mathrm{~cm}$.

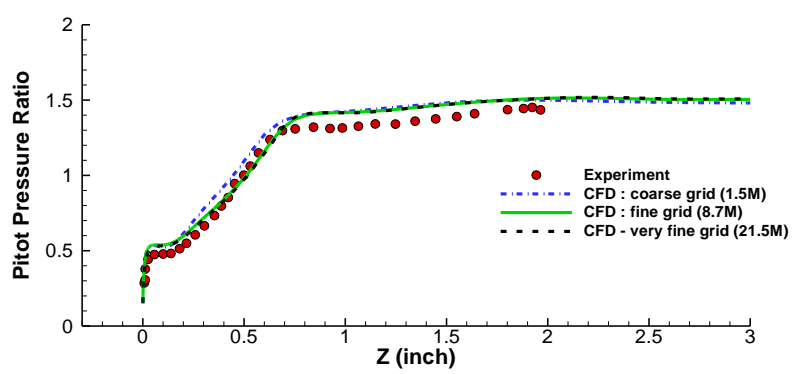

(b) $y=5.22 \mathrm{~cm}$.

Fig. 3: Comparision of computed and experimental pitot pressure distribution at $x=12.95 \mathrm{~cm}$

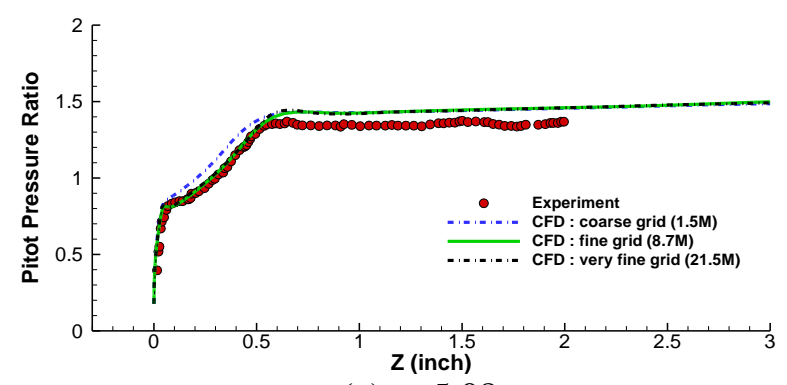

(a) $y=5.08 \mathrm{~cm}$

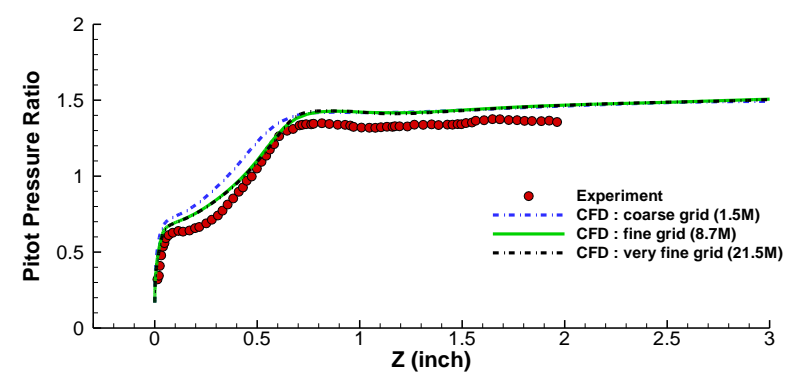

(b) $y=6.35 \mathrm{~cm}$

Fig. 4: Comparision of computed and experimental pitot pressure distribution at $x=18.03 \mathrm{~cm}$

The stream-wise coordinate $X$ is measured from the leading edge of the shock generator, the normal coordinate $Y$ is measured away from the tunnel surface where wedge is mounted, and the $Z$ coordinate is normal to $X-Y$ plane. The grid and boundary conditions used for CFD analysis are presented herein. Half of the wind tunnel was modeled in the present work with coarse grid size of $298 \times 81 \times 65$, see Fig. 2(a). The inflow velocity profile of turbulent boundary layer was provided by computing a square duct to the point that the boundary layer thickness approximately matching the thickness of Oskam's experiment. Symmetry was assumed in the transverse $\mathrm{Z}$ direction in the computation. Note that the bottom wall here refers to the wall where the incident shock generated from the wedge impinges, in order to follow the conventional definition. Similarly, the wall neighboring the wedge and the bottom wall is named the side wall. Several numerical studies have been conducted in the past ${ }^{13,17,18}$.

Fig. 2(b) shows the experimental survey planes along with the shock generator. These planes are the same as 
in the CFD analysis for the purpose of comparisons. The comparisons with experimental data in terms of pitot pressure ratio through the boundary layer in the $Z$ direction at rake locations of $x=12.95$ and $18.03 \mathrm{~cm}$ are shown in Figs. 3 and 4 respectively. The pitot pressure is the ratio of local pitot pressure to the freestream pitot pressure. The transverse gradients in pitot pressure ratio near the sidewall $(z=0 \mathrm{~cm})$ shown in Fig. 3 (a)-(b) and Fig. 4(a)-(b) are attributed to the interaction of the shock wave with the tunnel sidewall boundary layer. The computed result agrees better with the experimental data near the wall and overpredicts at the outer portion of the boundary layer.

To assess the effects of grid refinement on the solution, three grids of different coarseness have been used for the baseline simulation described in this work. All three grids have the same spacing in the streamwise direction, but of various refinement in the other directions. For the coarse grid, its size is $298 \times 81 \times 65=1.5\left(\times 10^{6}\right)$ mesh cells. A fine grid of $298 \times 241 \times 121=8.7\left(\times 10^{6}\right)$ and a very fine grid of $298 \times 321 \times 225=21.5\left(\times 10^{6}\right)$ are also used. Their corresponding solutions are shown in Fig. 3 and 4 together with experimental data. As expected, the agreement between the solution and the data improves with the finer grid, especially near the wall. Note that both figures indicate solutions from the coarse grid are not accurate enough and little variation between the fine and very fine grids can be seen from the comparison. Thus, the fine grid system is chosen for the following aerodynamic analysis. Its minimum mesh spacing of $0.0005 \mathrm{~mm}$ is enforced at the wall. A detailed flow analysis will be presented in section IV.

\section{Optimization of Controlling SWBLI in Supersonic Inlet}

Design processes include defining objective functions, setting up the geometry representation, and searching for optimal geometry using optimizer. Each process and its relevant information are introduced in the subsections below.

\section{A. Objective function}

Three objective functions are considered in this study. Two are traditional performance metrics: total pressure recovery, averaged circumferential distortion descriptor ${ }^{20}$. The third one is a newly derived objective function which locates the region of the separated flow and is named separation function in this paper. In the present study, 2-D problems adopt total pressure recovery and separation function while 3-D problems use total pressure recovery and distortion. These objective functions are defined as follows;

a. Total pressure recovery $(P R)$ :

Total pressure recovery is a primary inlet performance parameter and is defined as the area-averaged ratio of the total pressure at inlet exit $\left(P_{t_{e}}\right)$ and the free-stream total pressure $\left(P_{t_{0}}\right)$,

$$
P R=\frac{P_{t_{e}}}{P_{t_{0}}}
$$

b. Separation function $\left(S E P_{\text {avg }}\right)$ :

To address the weak boundary layer and flow separation caused by SWBLI, the local skin friction distribution, $C_{f x}(x)$, is chosen. The skin-friction coefficient is a good indicator of the condition of the boundary layer. When flow separates, the zero $C_{f}$ value indicates the onset of separation; it continues being negative for reverse flow until $C_{f}$ value becomes zero again when flow reattaches. Thus, the location and size of flow separation bubble can be identified and accordingly, optimization can be applied to remove or at least delay the flow separation. Moreover, the weak boundary layer and the incipient separation can also be indicated by positive but small $C_{f}^{19}$. A separation function is defined in the following,

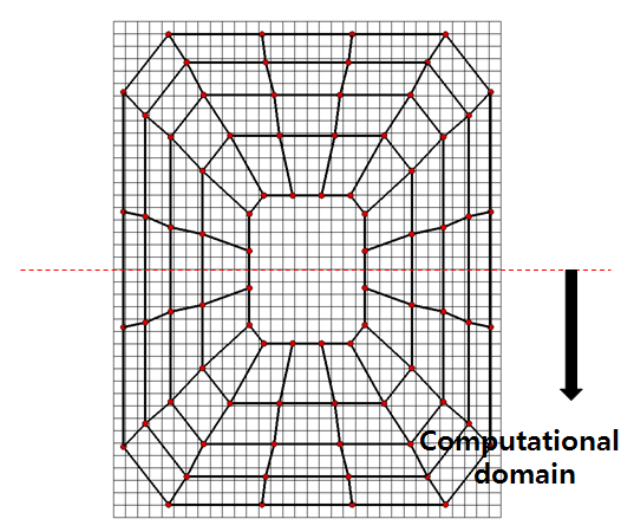

Fig. 5: Area weighted AIP rake for the measurement of distortion.(installed at $\mathrm{x}=55 \mathrm{~cm}$ ) 


$$
\begin{aligned}
& S E P_{\text {avg }}=\frac{\int S E P(x) d A}{\int d A} \\
& \text { where, } \quad \operatorname{SEP}(x)= \begin{cases}1.0 \quad\left(\text { if } C_{f x}(x)>C_{\text {cutoff }}\right) \\
C_{f x}(x) / C_{\text {cut-off }} & \text { (otherwise })\end{cases}
\end{aligned}
$$

The concept of $\operatorname{SEP}(x)$ is to ensure the optimizer recognizes only the weak boundary layer or separated flow regions where $C_{f x}(x) \leq C_{\text {cut-off }}$ during the design process. Here, $C_{\text {cut-off }}$ is set based on the flow analysis result of the baseline model.

c. Averaged circumferential distortion descriptor ${ }^{20}\left(\mathrm{DPCP}_{\text {avg }}\right)$ :

Inside a supersonic inlet, a thick boundary layer, shock wave, secondary flow, or flow separation involve energy losses. Energy loss leads to a reorientation of the flow fields which in turn results in distortion. Thus, at the exit of inlet, a different pressure inlet performance parameter can be characterized by distortion, as shown below,

$$
D P C P_{\text {avg }}=\sum_{i=1 . .5} \frac{1}{5}\left(\frac{P A V_{i}-P A V_{\text {LOWi }}}{P A V_{i}}\right),
$$

where $i$ is the ring number on the inlet exit plane or AIP rake, $P A V_{i}$ is the area averaged total pressure of the ring $i$ and $P A V_{L O W i}$ is the averaged low total pressure sector of the ring $i$. Following the conventional area weighted averaging in the circular duct, a schematic display of the area weighted averaging in a rectangular duct is presented in Fig. 5.

\section{B. Geometrical representation: shape parameterization}

The design space for the tailored perturbations on the bottom wall where the viscous layer interacts with the incident shock wave is predetermined based on the inviscid flow theory for the purpose of reducing the number of design variables. The surface geometry is represented by a patch which is described by the non-uniform rational basis spline (NURBS). The local coordinate $\mathbf{S}(u, v)$ of the patch can be mapped out from the global coordinate $\mathbf{P}(x, y, z)$. Figure 6 depicts the NURBS surface modeling the design space (domain of interest) used in this study. A NURBS patch is defined by the order of basis functions, a set of weighted control points, and a knot vector. The control points $\left\{\mathbf{P}_{\mathrm{i}, \mathrm{j}}\right\}$ determine the curve/edge while the surface is computed by taking a weighted sum of a numbers of control points; the weight varies with the basis functions used and decreases smoothly to zero. The knot vector contains a sequence of parameter values and it divides the parametric space into intervals referred to as knot spans.

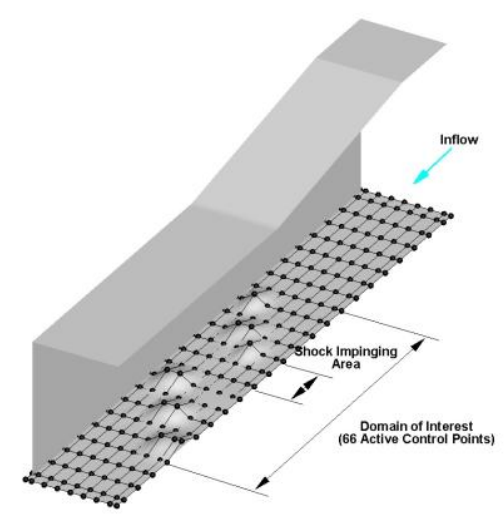

Fig. 6: A schematic of aNURBS surface patch representing the deformation of a duct wall Each time the parameter value enters a new knot span, a new control point becomes active, while the old control point is disabled. The order of a NURBS surface defines the number of nearby control points that influence any given point on the surface. Similarly, a NURBS patch is situated on the side wall where the glancing SWBLI occurs. The sidewall patch extends from the leading edge to the exit. Thus, a larger number of control points are needed than the bottom wall

\section{Optimization Method}

An evolutionary multi-objective algorithm, NSGA-II, is chosen for its convergence speed and its ability to control crowding and to obtain solution diversity ${ }^{17}$. The details in its constrained tournament selection through crossover and mutation operations and how to introduce random changes in each generation are discussed in 
Ref. 17 and defaults are used in this study. In the multi-objective optimization, NSGA provides a pareto front, or set of non-dominating potentially optimal solutions in design space. Although NSGA-II is known for its speed, it is still very computationally expensive when compared with gradient-based adjoint method, especially when a large number of design variables are considered. In order to deal with the expensive computing cost, a task parallel process using MPI and GNU parallel is implemented to combine the CFD code and NSGA-II. As a result, all individuals at each generation are solved in parallel. In addition, the spatially high-order schemes generally require more number of iterations or sometimes fail to converge. Thus, the 3-D problems are solved using $1^{\text {st }}$ order spatial accuracy during the optimization while the performance evaluation of optimal solutions is carried out with high-order accuracy. If there is any occurrence of non-converged CFD solution during the flow analysis of the individual, large numerical values are assigned to objective functions to drive the optimizer away from that design space.

\section{Results and Discussion}

\section{A. Maximizing separation function : 2-D case, single objective}

A two-dimensional test case with prescribed shock conditions on a turbulent flat plate, as shown in Fig. 7 is used to investigate the concept of geometric shaping for the negotiation of the shock induced-separation. The mesh employed is $341 \times 139$. The grids are highly clustered around the shock impinging point to maintain highresolution around the perturbed surface region.

The objective function considered is the separation function. The number of design parameters is 3 which are uniformly distributed at $35 \mathrm{~cm} \leq x \leq 42 \mathrm{~cm}$. There are 48 individuals are used and 48 generations are set for NSGA-II. The domain of computation is defined by $-9 \mathrm{~cm} \leq x \leq 80 \mathrm{~cm}$ and $0 \mathrm{~cm} \leq y \leq 20 \mathrm{~cm}$. The incoming boundary layer profile with thickness $(\delta)$ of $1 \mathrm{~cm}$ is obtained from turbulent flow over a flat plate and is interpolated at $x=-9 \mathrm{~cm}$. Instead of using shock generator of a $10^{\circ}$ deflection angle, an equivalent shock strength is generated by imposing the inviscid pre- $(x<0 \mathrm{~cm})$ and post- $(x$ $\geq 0 \mathrm{~cm}$ ) shock conditions as the boundary condition at the upper boundary $(y=20 \mathrm{~cm})$ for flow with freestream Mach number of 2.89. The blue zone indicates pre-shock condition and green for post shock, as seen in Fig. 7.
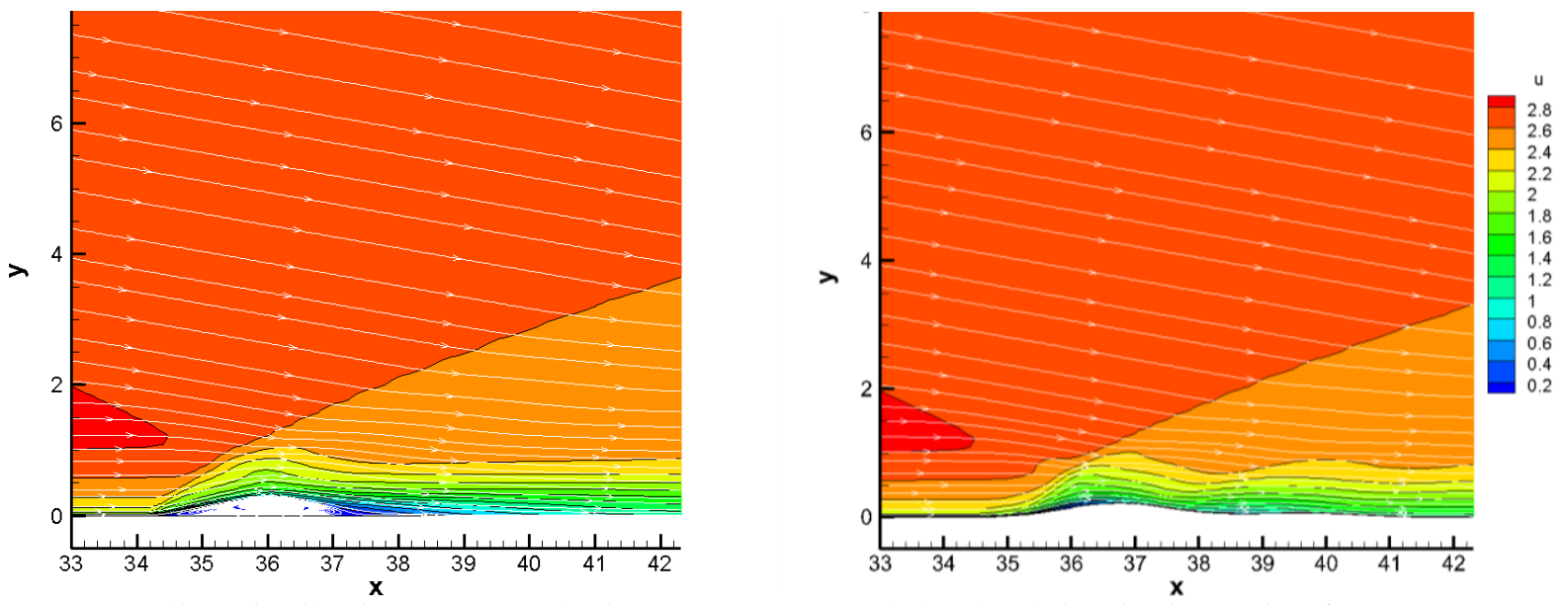

Fig. 8: Streamline distribution over $x$-velocity contours around the shock impinging point for baseline (Left) and optimized geometries (Right). 
Figure 8 shows the flow streamlines over the $x$-velocity contours near the shock-impinging region and the skin friction coefficients against axial direction $x$, baseline case on the left and optimized one on the right. The thinning blue region in the optimal design on the right when compared with the baseline $x$-velocity contours indicates that the separation is removed. In Fig. 9, the distribution of skin friction coefficient confirms that the area of the negative skin friction coefficients is fundamentally alleviated so that the "health" of the boundary layer is improved remarkably. It is observed in the optimal design that a convex bump appears very close to the baseline separation bubble (roughly $1 \mathrm{~cm}$ behind). Instead of separating, the flow accelerates and expands at the onset of the bump in the flow in the optimal geometry. Apparently, this change of shape through optimization technique can significantly improve the separation in 2-D flow. However, the fixed configuration approach may have a shortcoming in its susceptibility to deteriorating performance due to variation in flow conditions. To address this problem, the next multiple design points study is conducted.

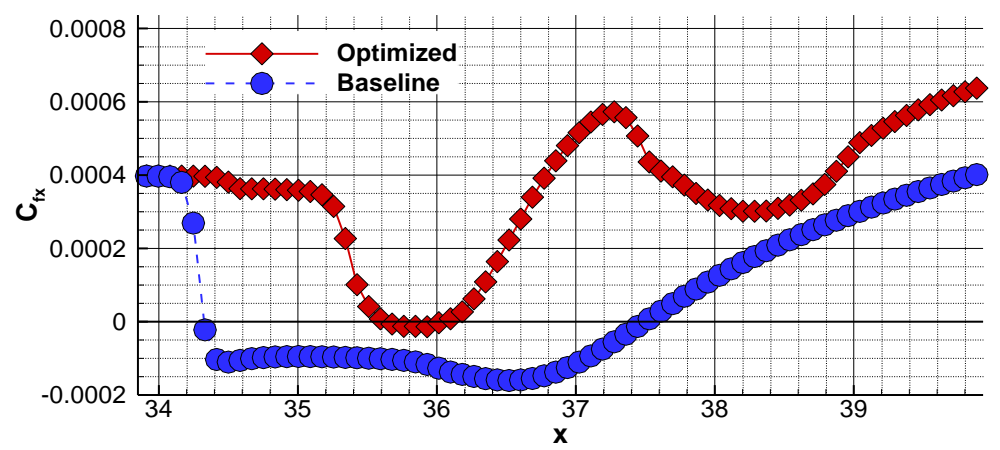

Fig. 9: Comparison of skin friction coefficient distribution around the shock impinging point between optimized geometry and the baseline.

\section{B. Multi-point design: 2-D case, multi-objective}

The supersonic flow field may be inherently highly sensitive to even a very small perturbation in the shape or boundary conditions because of the high flow speed and the compressibility. As a result, a design which shows noticeably improved performance at the design condition may not do well in other off-design conditions. In order to achieve robust optimized shapes that can sustain good performance over a wide range of flow conditions, thus, as an extension of the above single objective study, a multi-point multi-objective design case is examined in this section. A 2-D supersonic inlet is designed to operate at two operating conditions $(\mathrm{M}=2.5$
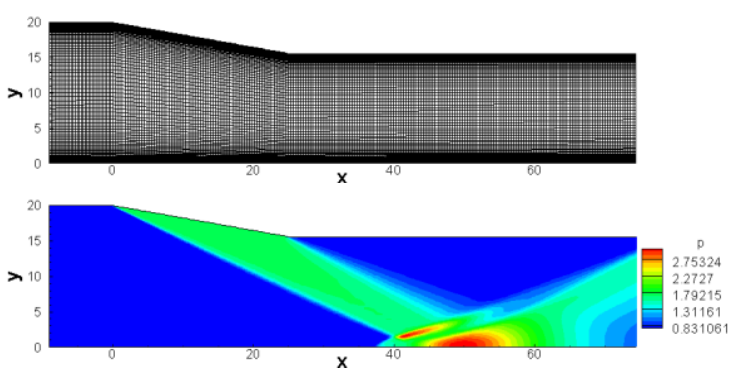

Fig. 10: Computational domain and pressure distribution at $\mathrm{M}=3.5$ and $\mathrm{M}=3.5$ ). After performing geometric optimization on this inlet corresponding to each condition, these two resulting geometries will be superposed together to be tested at both operating conditions. The baseline geometry used in the study is shown in Fig. 10. The wedge/shock generator angle is $10^{\circ}$ and the grid size is $247 \mathrm{x} 161$. The objective functions considered are total pressure recovery and separation function.

The proof that the optimal shapes from $M=2.5$ and $M=3.5$ conditions are capable of eliminating separation successfully can be seen in figures 11 and 12 for both conditions respectively. The reduction of separated flow is reflected in the gain in pressure recovery by around $1.5 \%$ and $1.7 \%$ for each case as presented in Tables 1 and 2 . Figure 11 shows the comparison of streamlines and Mach contours between baseline and optimized geometry at $\mathrm{M}=2.5$. As observed in the previous $\mathrm{M}=2.89$ case, optimal design shows a convex bump at similar position. Thus, the flow accelerates and expands at the onset of the bump as well. This results in a thinner boundary layer thickness in the downstream and consequently weakens the following compression waves (reflecting shock) near the surface because of the expansion on the peak of bump. The pattern is similar in the design at $M=3.5$, given in Fig. 12, but the height of the bump is lower than that of lower speed cases because the size of the separation 
bubble in the baseline is smaller. It is also shown that the front part of bump rises gradually toward the downstream direction so that the loss from the abrupt blockage effect by the bump is diminished through the process of optimization.
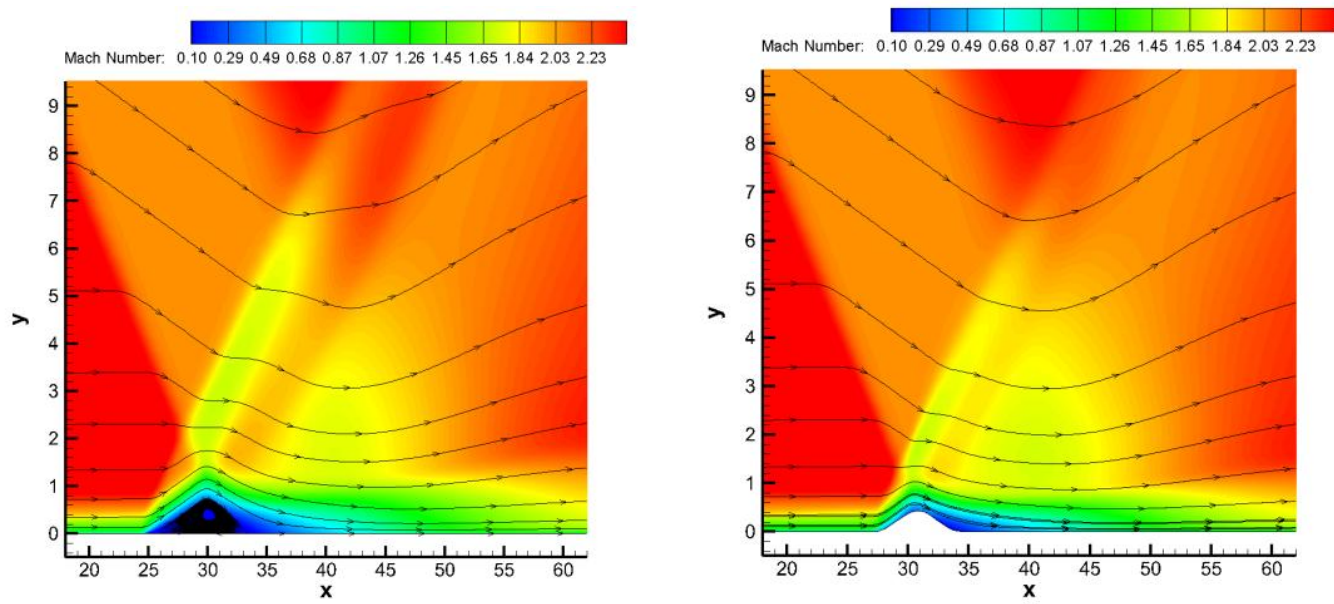

Fig. 11: Streamline distribution over Mach contour around the shock impinging point for $M=2.5$ cases, including baseline (Left) and optimized geometries (Right: Best separation function)

Considering the geometric difference between best separation function and best PR cases, the difference of objective functions is almost negligible and the skin friction distribution given in figure 13 confirms that the PR and separation function are not in a trade-off relation. Thus, the gains in separation function are proportionally reflected in the improvement of pressure recovery in the current 2-D studies.
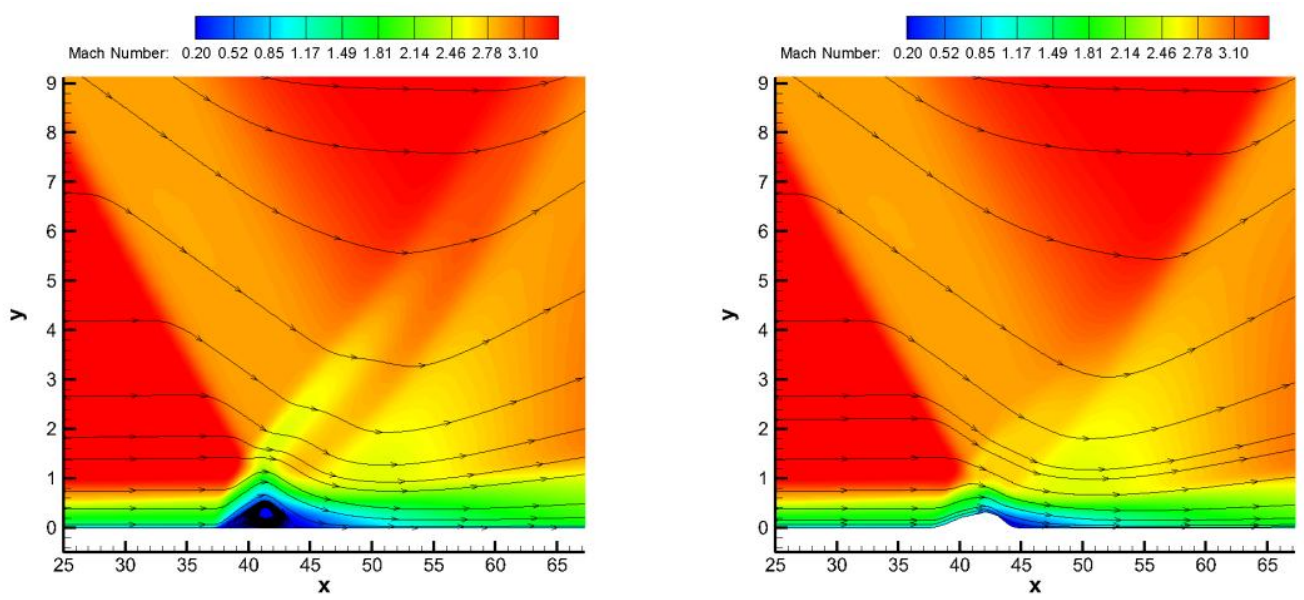

Fig. 12: Streamline distribution over the Mach contours around the shock impinging point for $M=3.5$ cases, baseline (Left) and optimized geometries (Right: Best PR)

Table 1: Multi-objective optimization solutions in comparison with the baseline performance. (M=2.5)

\begin{tabular}{|l|c|c|}
\hline \multicolumn{1}{|c|}{ Case } & Total Pressure Recovery, $P R$ & Averaged Separation Function, $S E P_{\text {avg }}$ \\
\hline Baseline & 0.8685 & 0.8251 \\
\hline Best $P R$ & 0.8832 & 0.9125 \\
\hline Best $S E P_{\text {avg }}$ & 0.8822 & 0.9153 \\
\hline
\end{tabular}


Table 2: Multi-objective optimization solutions in comparison with the baseline performance. $(\mathrm{M}=3.5)$

\begin{tabular}{|l|c|c|}
\hline \multicolumn{1}{|c|}{ Case } & Total Pressure Recovery, $P R$ & Averaged Separation Function, $S E P_{\text {avg }}$ \\
\hline Baseline & 0.8227 & 0.8654 \\
\hline Best $P R$ & 0.8395 & 0.9598 \\
\hline Best $S E P_{\text {avg }}$ & 0.8382 & 0.9648 \\
\hline
\end{tabular}

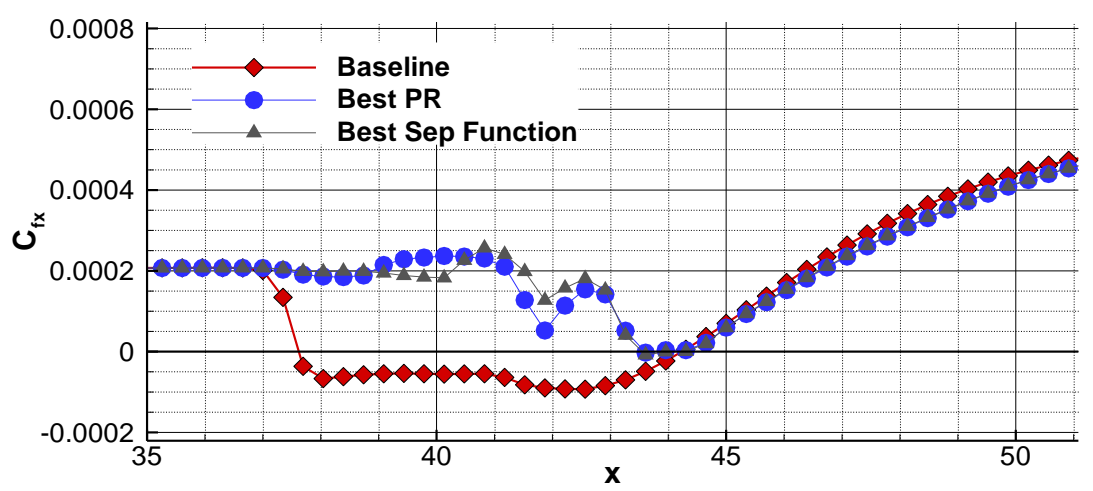

Fig. 13: Comparison of skin friction coefficient distribution around the shock impinging point between baseline and optimized cases of $\mathrm{M}=3.5$.

Table 3: Off-design condition performance of superposed geometry at $\mathrm{M}=3.5$ and $\mathrm{M}=2.5$.

\begin{tabular}{|c|c|c|c|}
\hline \multirow{2}{*}{ Geometry } & Condition $(M)$ & $\begin{array}{c}\text { Total Pressure } \\
\text { Recovery, } P R\end{array}$ & $\begin{array}{c}\text { Averaged Separation Function, } \\
\text { SEP }\end{array}$ \\
\hline \multirow{2}{*}{ Baseline } & 2.5 & $\mathbf{0 . 8 6 8 5}$ & $\mathbf{0 . 8 2 5 1}$ \\
\cline { 2 - 4 } & 3.5 & 0.8227 & 0.8654 \\
\hline \multirow{2}{*}{ Superposed } & 2.5 & $\mathbf{0 . 8 5 5 7}$ & $\mathbf{0 . 7 8 5 2}$ \\
\cline { 2 - 4 } & 3.5 & 0.8247 & 0.9392 \\
\hline
\end{tabular}
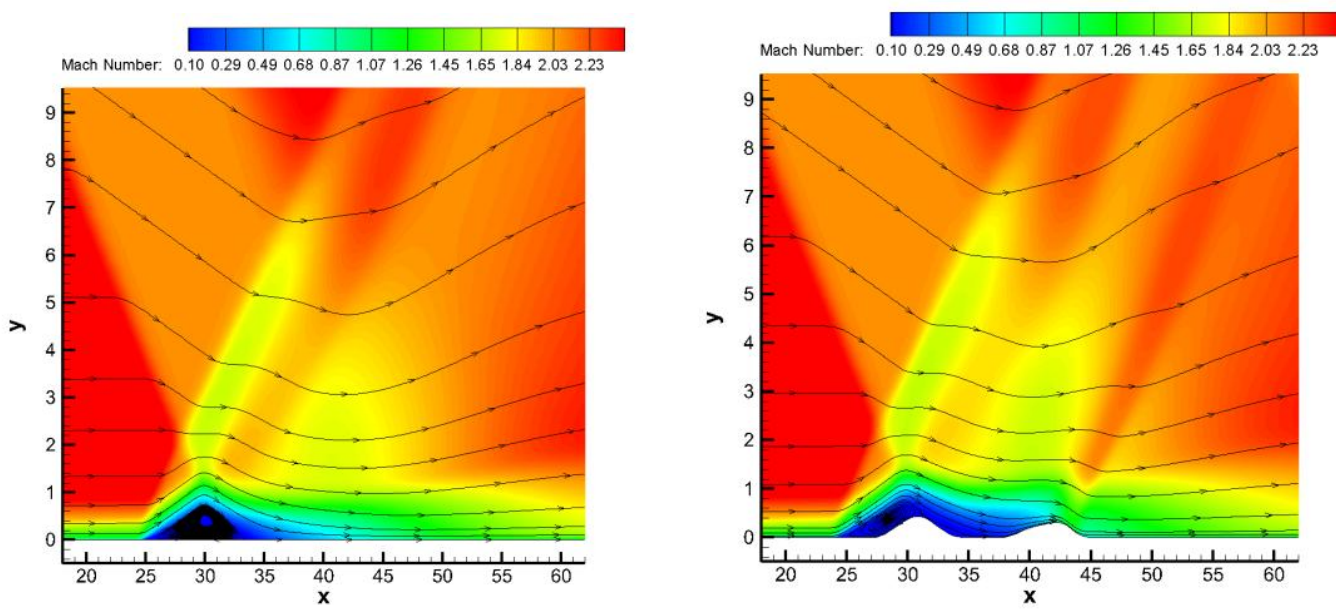

Fig. 14: Streamline distribution over Mach contours around the shock impinging point at $M=2.5$, baseline (Left) and superposed design (Right)

The present study considers separation control with micro-scale surface shaping so that a geometry designed in one condition may not degrade the performance in other conditions. Thus, for the examination of robustness of the optimized geometries in the performance metric, the two designs which are investigated in different conditions are superposed and are tested in both design conditions. 
Table 3 presents the performance of the superposed geometry compared with the baseline geometry at $\mathrm{M}=2.5$ and $M=3.5$. In the case of $M=3.5$, the superposed geometry maintains its performance superiority to the baseline geometry but it shows poorer performance than at design point which is summarized in Table 2. In addition, the superposed one shows a worse performance at $\mathrm{M}=2.5$ than the baseline geometry does. Figure 15 depicts how the shock and boundary layer evolve at $\mathrm{M}=2.5$ after the shock-impingement on the superposed geometry. Thus, even if the separation could be controlled by the first bump, the influence from the second bump has a detrimental effect by increasing the boundary layer thickness at the first bump.

Based on these observations, it can be concluded that the micro-scale shaping may bring positive effect to increase total pressure recovery remarkably in two dimensional SWBLI. To demonstrate the robust operability under various conditions, more powerful optimization techniques are needed to incorporate all objectives at all design points, such as a multi-objective optimization methods. Further study is required.

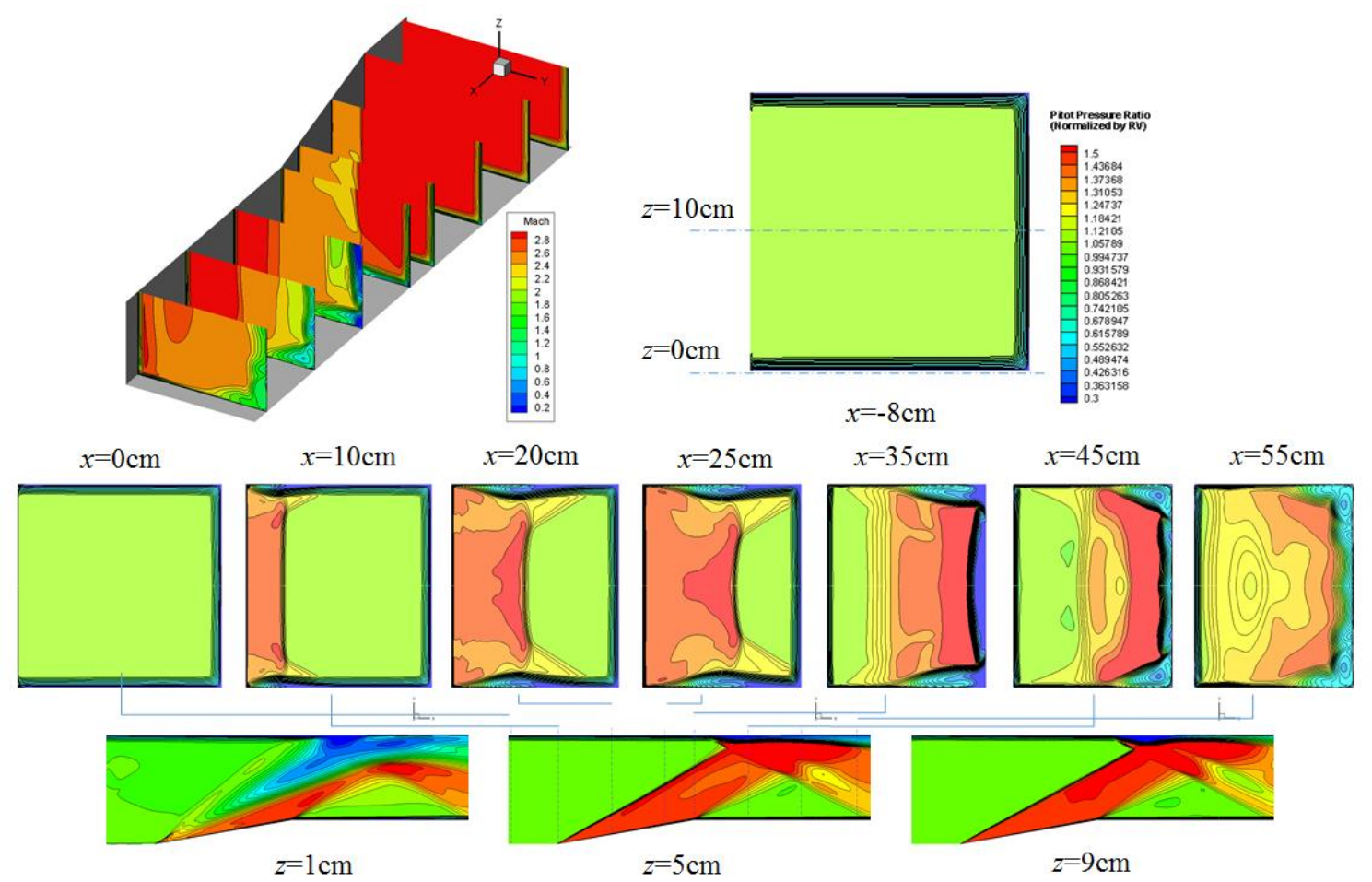

Fig. 15: Mach number and pitot pressure contours of flow-fields displayed at various stream-wise and span-wise planes. (a) top-left: cross-section Mach contours along various $x$-locations; (b) top-right: inflow boundary layer thickness in the pitot pressure contour;

(c) middle: sectional pitot pressure contours at various $x$-locations; (d) bottom: pitot pressure contours at various $z$-locations where $z=10 \mathrm{~cm}$ is the symmetry plane.

\section{Maximizing total pressure recovery and minimizing $D P C P_{\text {avg }}: 3-D$ case, multi-objective}

The CFD analysis of the baseline flow-field, i.e., one without flow control, has been validated with experimental data as described in section II. Fig. 15 presents the Mach number contours at various stream-wise and span-wise planes. Figure 15-(a) shows the Mach contours in various cross sections along the $x$-direction. Figure 15-(b) displays the inflow boundary layer thickness in terms of pitot pressure contours used in the computation and 15-(c) depicts pitot pressure contours at various stream-wise planes. It also demonstrates how the oblique shock wave from the wedge sweeps along the sidewall. The imposition of the static pressure gradient upon the low momentum near wall viscous flow results in sidewall vortex and subsequently a lateral migration of the subsonic portion of the sidewall boundary toward the corner. As explained in Ref. 13, this sidewall vortex elongates and grows stronger in the downstream $x$-direction, seen on planes from $x=10 \mathrm{~cm}$ to $35 \mathrm{~cm}$ in Figs. 15(c), resulting in low energy fluid accumulated in the corner and eventually a corner separation. Also, separation 
in the sectional pitot pressure contours at the stream-wise location of $x=35 \mathrm{~cm}$ is observed in 15 -(c). Figure $15(\mathrm{~d})$ shows the side wall influence diminishing in the coordinate $z$-direction, i.e. from $z=1 \mathrm{~cm}$ to $z=9 \mathrm{~cm}$ (close to core flow). Oil flow pattern on the side and bottom walls is presented in Fig. 16, and the axial velocity at the first mesh point off the wall is plotted in contour levels of negative and positive values in the same figure. Regions of reverse flow seen in green in Fig. 16 are observed on the bottom wall where the incident shock impinges at and in the corner where the bottom wall meets sidewall. This corner separation appears first, which is also observed experimentally ${ }^{22}$, and originates at $x=22 \mathrm{~cm}$ and extends some finite axial dimension in the downstream direction to about $x=38 \mathrm{~cm}$. The bottom wall separation is located close to the centerline at the symmetry plane and originates roughly at $x=29 \mathrm{~cm}$ and reattaches downstream at $x=38 \mathrm{~cm}$. Figs. 15 and 16 together depict how the baseline flow-field behaves under three kinds of oblique SWBLI. An incident shock occurs where the wedge shock intersects the bottom wall. Side wall SWBLI results in streak lines being converged following the shock angle and bent toward the intersecting bottom wall when the shock from the wedge sweeping along the sidewall. In the corner, secondary flows are formed and separated by the adverse pressure gradient due to the side wall SWBLI.

The separation originating from the bottom wall which is also named central separation exhibits a 2-D separation characteristics while the corner separation is three-dimensional and induced by the SWBLI at the sidewall $^{21}$. Subsequently, the corner separation can be seen as a corner blockage which generates conical shock wave. Therefore, the impinging shock, the reflected shock, the corner shock and their associated separations interact and intercept one another in this complicated flow-field. It is observed that the corner separation is induced by the viscous sidewall and its glancing sidewall SWBLI. In Fig. 16, those streamlines near the bottom wall turn at the point A toward the center line when flow crosses the conical shock wave. The reverse flow from the corner separation also turns to the center region of the bottom floor. Because of the three dimensional effect, a secondary flow originating from the corner reverse flow rolls up toward the center of the bottom floor, starting from the region between A and E. It intersects with the incident shock from the wedge, thus forms a separation bubble rotating counter-clockwise on the $x-z$ plane centered at the point B. Now shape of the central separation is changed, not only perpendicular to the bottom floor as observed in 2-D problems but also transversely conforming to the above described much larger counter-clockwise rotational bubble. From these observations, it can be deduced that the energy loss from the separation induced directly by the incident shock impingement is much smaller than what is caused by the 3-D effect. It is due to that the flow features in the present test case are mostly dominated by the corner separation and the secondary flow. Also, Fig. 16 demonstrates the strong coupling of corner separation and central separation.

We are interested in uncovering the mechanism whereby geometric elements in the rectangular duct, i.e. the bottom, the side and corner, exert their influence to the flow's aerodynamic characteristics. With specific emphases on addressing three types of SWBLI and mitigating their adverse effects in aerodynamic performance, bottom and side geometries will be perturbed, and then optimized separately in this study. In addition, a simple change of the corner shape by filleting is used to demonstrate the importance of the corner effect in the 3-D SWBLIs. Following our two-dimensional geometric optimization procedure, the three dimensional wall shaping which is defined by the optimizing objectives for the best pressure recovery and least flow distortion are described and discussed next. 

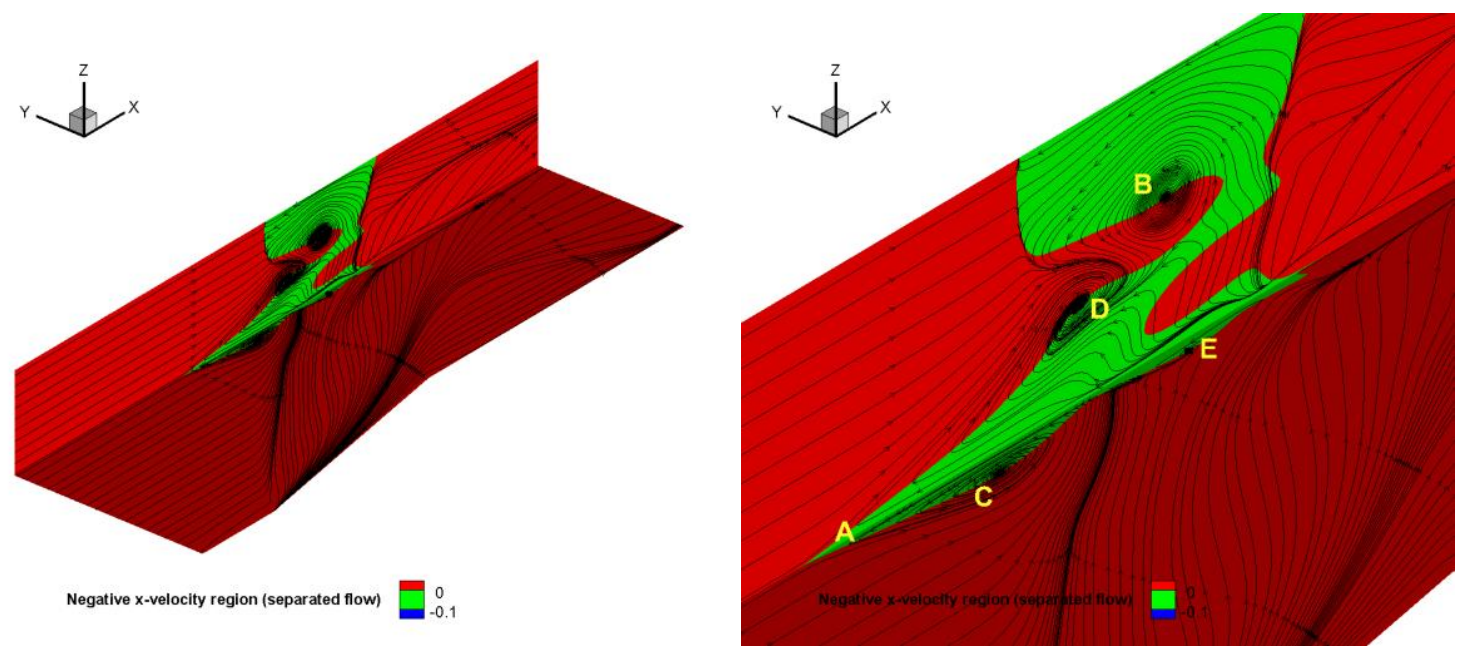

Fig. 16: Oil flow pattern on the bottom and side walls and in corner region.

The optimization is carried out by constructing NURBS patches on the design domains of interest at the bottom and side walls. Their corresponding control points are determined based on the inviscid flow theory. Note that the perturbation at each control point can only vary within $10 \%$ of the inflow boundary layer thickness in the following studies, unless it is specified otherwise.

\section{Incident and reflected shocks - bottom floor design}

Deducing from the flow characteristics of the baseline duct geometry, the effect of shock-induced separation from SWBLI on the bottom surface is one of the reasons for a low quality of incoming flow ingested at API. Considering the bottom wall only, a pre-developed thick boundary layer on it separates due to the adverse pressure gradient caused by the shock wave from the wedge. Furthermore, this central separation is also influenced by the vortical flow and separation at the corner. For the first design case, the bottom wall shaping is tested to evaluate how the shock-induced separation can be controlled by the optimization.

The total number of control points for the bottom wall is 66. They are positioned ahead and in the rear of the shock-impinging area as shown in Fig. 7. The objectives are to minimize the distortion and to maximize the total pressure recovery. The population number of each generation is 48 and the number of the generation is 60 due to a prohibitive computational

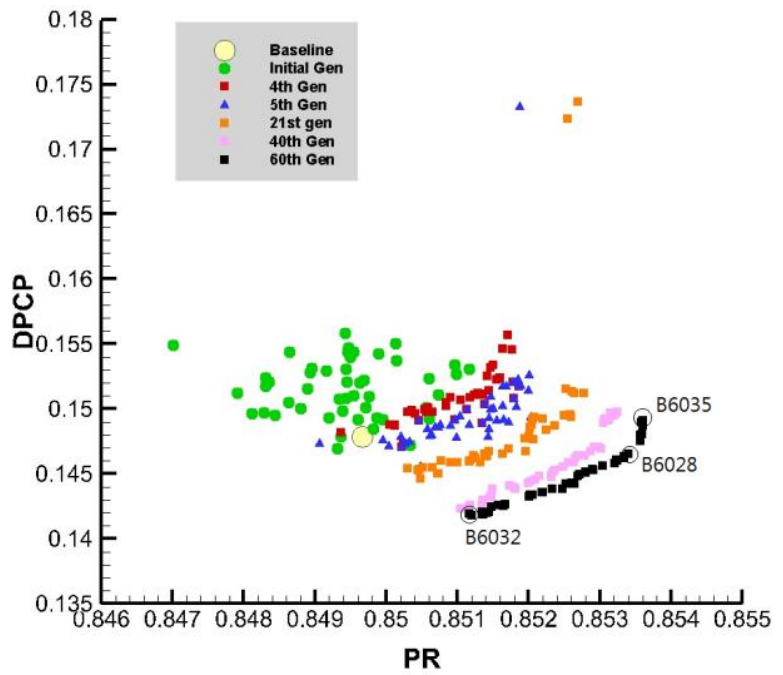

Fig. 17: Pareto front of the bottom floor design, where B6032- the least $\boldsymbol{D P C P}, \mathrm{B} 6035$ - the best PR and B6028 - balanced. 


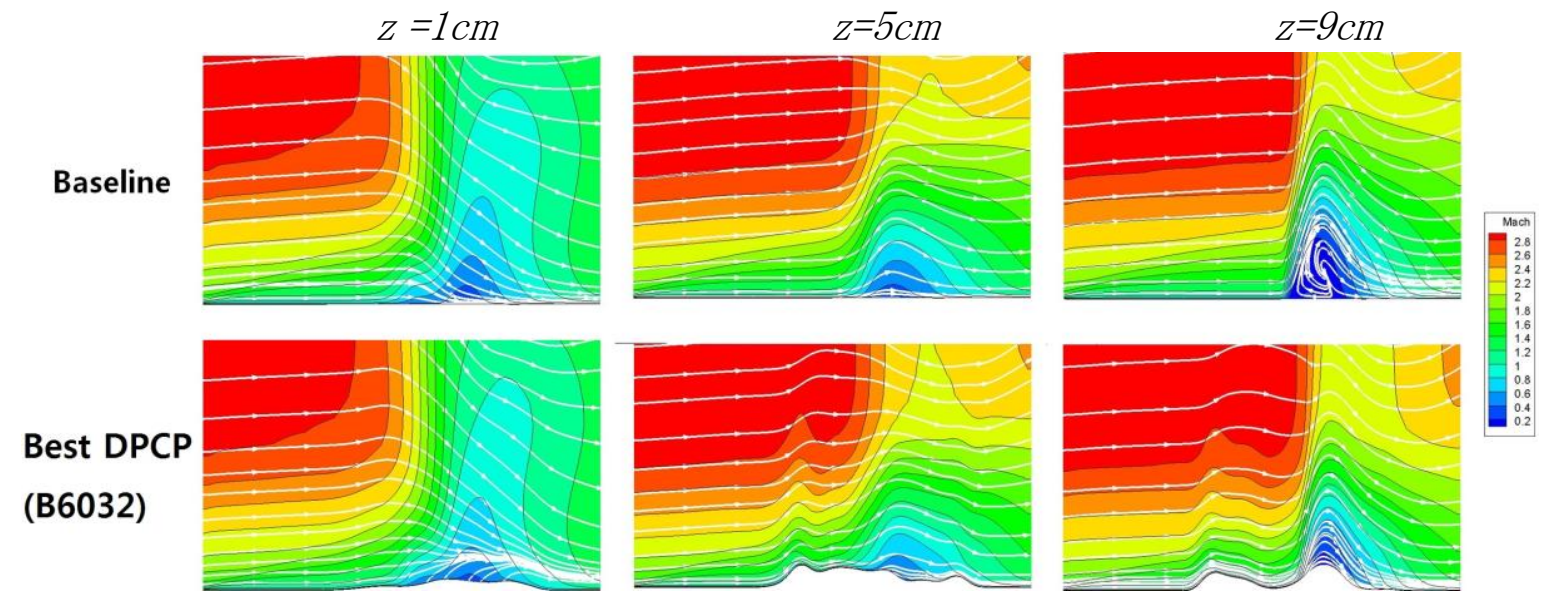

Fig. 18: Comparison of geometric change, Mach contours and streamline patterns (select zconstant sections, Note that the scale ratio of $x$ to $y$ coordinates are 1:25 to magnify the flow pattern and geometric changes; the contour covers whole computational domain along the streamwise direction $x:-9 \mathrm{~cm} \leq x \leq 57 \mathrm{~cm}$, and viscous flow area along perpendicular direction $y: 18 \mathrm{~cm} \leq y$ $\leq 20 \mathrm{~cm}$ (where $\mathrm{y}=20 \mathrm{~cm}$ is the bottom wall location).

Figure 17 shows the Pareto front of the bottom wall design. The Pareto front gradually forms as the generation goes over from the randomly distributed initial individuals in Fig. 17. It also shows the trade-off relationship between two objectives. It is obvious that the overall performance evolves and performance is improved as the optimization converges. At the $60^{\text {th }}$ generation, the geometric change for the minimization of the $\boldsymbol{D P C P}$ as well as maximizing the total pressure loss as the Pareto front spreading wider. The fact that $60^{\text {th }}$ generation shows better but no significant variation from the $40^{\text {th }}$ indicates the solution at $60^{\text {th }}$ is close to the converged solution. However, the improvement from the yellow circle which represents the baseline looks very limited, i.e., overall, the distortion is reduced by less than $3 \%$ and the total pressure recovery increased by no more than $0.5 \%$. In addition, some of the best pressure recovery performers show almost no improvement in the distortion performance from the baseline.

Among all points on the Pareto front, we select a best performer for least distortion to investigate the effect of bottom floor shaping. Table 1 presents the comparison of performance metrics between baseline and the optimized geometry (B6032).

The optimized geometry shows higher pressure recovery and lower distortion performance simultaneously against the baseline model. As a consequence, the chosen best performer is investigated individually to understand how the bottom wall shaping improves the performance through Mach contours and separation function distributions in the following comparison.

Table 4: Comparison of performance metric for bottom floor design ( $1^{\text {st }}$ order values $)$

\begin{tabular}{|c|c|c|}
\hline Case & $\begin{array}{c}\text { Total Pressure Recovery } \\
(\text { PR })\end{array}$ & $\begin{array}{c}\text { Distortion Coefficient } \\
\text { (DPCP) }\end{array}$ \\
\hline Baseline & 0.8496 & 0.1480 \\
\hline B6032 (Least DPCP) & 0.8512 & 0.1423 \\
\hline
\end{tabular}

Fig. 18 displays sectional views of Mach contours, stream-lines for the baseline and optimized models at selected span-wise coordinate $y$. The baseline model shows a strong shock-induced separation around the core flow region $(z=9 \mathrm{~cm}$, symmetric plane: $z=10 \mathrm{~cm})$. The optimized geometry shows that the wall shaping induces a significant reduction of separation at this region, having almost no reverse flow after shock-impinging point. Meanwhile the optimized design also depict that the near-wall flow is changed according to the change of bottom wall geometry from the baseline case. The overall trend of optimal geometric change is to generate a series of micro-scale bumps with gradually sloping peaks and valleys in the region where mild or strong adverse velocity profile is observed in the baseline flow-field. At $z=1 \mathrm{~cm}$ (close to side wall where $z=0 \mathrm{~cm}$ ), being different from the baseline case in which the flow close to side wall is bent laterally toward the bottom wall (i.e. 
y maximum), the optimized case indicates that the micro-bumps wall shaping causes nearwall streamlines being entrained together and pushed away from side wall by the blockage effect from these local bumps on the bottom wall. This specific local flow phenomenon caused by the right-angle corner disappears at locations of $z=5 \mathrm{~cm}$ and $z=9 \mathrm{~cm}$. The figures from $z=9 \mathrm{~cm}$ demonstrate this array of micro-bumps in $\mathrm{B} 6032$ energize the low momentum flows after shock-impingement by comparing the size of dark blue zones in B6032 and the baseline case. Thus, it agrees with what has been observed previously in 2-D optimization results that very low Mach number region caused by central separation can be minimized through the wall shaping optimization.
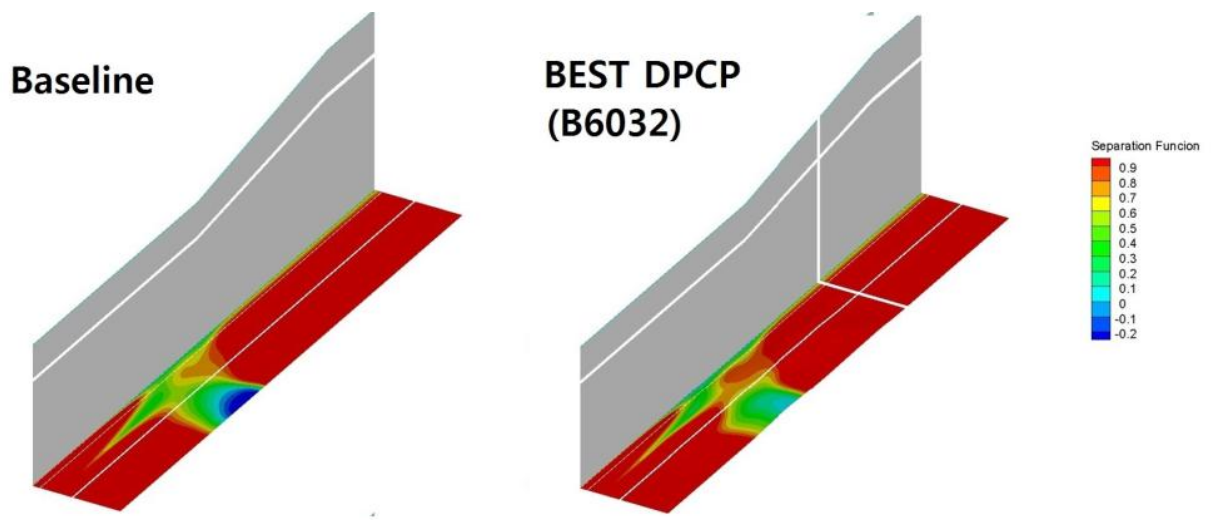

Fig. 19: Comparison of separation function distribution on the bottom floor obtained from the baseline and bottom wall design.

The plotted figures in Fig. 19 are the separation function distribution on the best $\boldsymbol{D P C P}$ and baseline geometry. The use of separation function in the present study is to locate the region of separation or the unhealthy velocity profile which leads to total pressure loss. Those non-red colored patches in the separation function contours indicate whereabouts of unhealthy boundary layer profiles ${ }^{19}$. The baseline does have the larger area of negative separation function around the symmetric plane, compared with that of the optimized design which shows almost diminished separation region. Deducing from the geometric change in Figs. 18 and the separation function distribution in Fig. 19, it can be concluded that the least distortion case takes advantage of wavy bump like geometry to disperse the low momentum flow, and subsequently the local strength of separation is diminished. Despite of clear indications that the bottom wall shaping can diminish the strength of the shock-induced separation from qualitative comparisons, the reflected improvement on the performance is merely $0.4 \%$ in the total pressure recovery and $4 \%$ in the distortion from the baseline case as indicated in Table 4.

As a result, the changes in total pressure contours at the outlet of the tunnel and the performance metrics through bottom floor design which contrarily expands the vortex flow in the corners are rather minor. Because of the strong coupling of the incipient separation on bottom wall and the corner separation as pointed out by Ref. 1, it is also found here that suppressing bottom wall separation may even degrade the corner separation, thus, the improvement in overall performance still remains limited.

\section{Glancing sidewall SWBLI - sidewall design}

The bottom floor design shows that a direct control of an incident shock induced central separation has insignificant effect on the performance metric when 3dimensional SWBLI is involved ${ }^{23}$. In the present and the following sections, geometric effects from sidewall and corner which distinguish 3-D problem from a 2-D SWBLI will be addressed. There are 209 design variables used in the sidewall design. These control points are uniformly distributed on the whole side wall

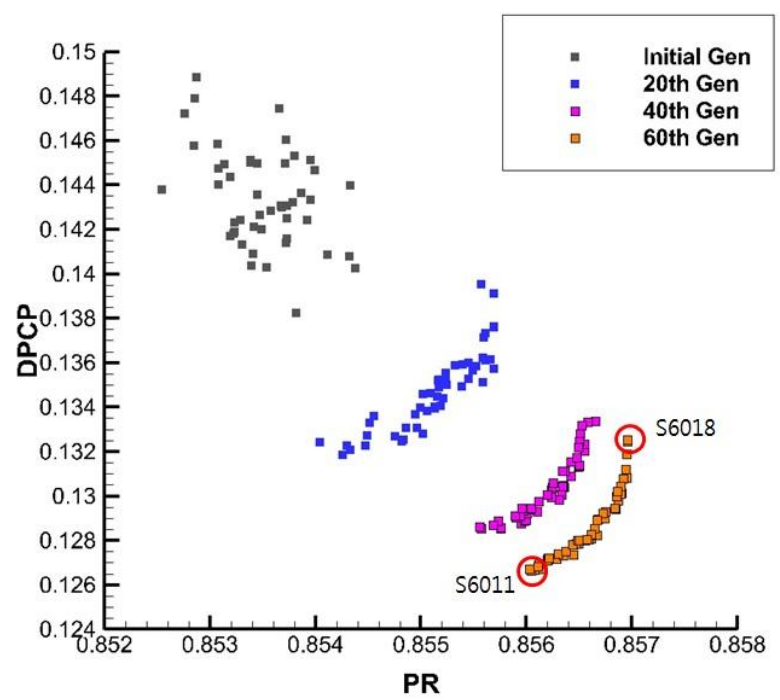

Fig. 20: Pareto front of the bottom floor design, where s6011 - least DPCP, s6018 - best PR. (Note that the history is represented by analyses from $1^{\text {st }}$ order spatial accuracy during the optimization.) 
domain and they are comprised by 19 (streamline- direction) by 11(span-wise-direction) grids, in a similar arrangement as those shown in Fig. 7 for bottom wall. The geometric constraints for the maximum geometric deviation are set to be less than $10 \%(-0.1 \mathrm{~cm} \leq \Delta z \leq 0.1 \mathrm{~cm})$ of the incoming boundary layer thickness $(\delta=1.37 \mathrm{~cm})$. The population size is 48 . The history of all individuals at various generations in design space and the corresponding Pareto front at the $60^{\text {th }}$ generation are presented in Fig. 20. Among these optimal individuals on the Pareto front, pitot pressure ratio contours at AIP station (i.e. the exit plane of the tunnel test section) of two best performers at the $60^{\text {th }}$ generation, i.e., the least $D P C P_{\text {avg }}$ (s6011) and the best PR (s6018) cases are compared with that of baseline (B0000) respectively in Fig. 21. The plots indicate an encouraging improvement from baseline case, i.e. the area of low pitot pressure region in the corner where bottom and side walls meet is reduced while the boundary layer on the side wall is a bit thicker. As a result the overall contour shows more circular and concentric shape, reducing the distortion. In addition, the area of highest pitot pressure region near the corner becomes larger so that the distortion is reduced by about $15 \%$ and pressure recovery is improved by around $0.3 \%$ even with a minor shape change (maximum $0.1 \mathrm{~cm}$ ) as presented in Table 5 . A notable thing is that the overall gains (in percentage) for both of the objectives from the optimization are maintained in the high order analysis. However, the best performers based on the $1^{\text {st }}$ order accuracy in each objective function could not remain superior to other individuals in the high order accuracy. We believe that it is because the differences in the performance and shape change between the best performers are not significant as observed in Fig.21.

Table 5: Side wall optimization solutions in comparison with the baseline performance. $\left(2^{\text {nd }}\right.$ order results)

\begin{tabular}{|l|c|c|}
\hline \multicolumn{1}{|c|}{ Case } & Total Pressure Recovery, $P R$ & $D P C P_{\text {avg }}$ \\
\hline B000000: Baseline & 0.8604 & 0.3248 \\
\hline s6011 & 0.8639 & 0.2842 \\
\hline s6018 & 0.8630 & 0.2761 \\
\hline
\end{tabular}

The oil flow pattern of the least DPCP case (s6011) and the area of reverse flow region, i.e., separated flow region, are presented and are compared with the baseline in Fig. 22. It can be seen that the size of the corner separation becomes a bit smaller in the least DPCP design, and thus a weaker corner shock and less interference with the central separation. As a result, the overall performance including distortion as well as the total pressure recovery is improved and it consistently supports the fact that the corner separation changes the size and shape of central separation. The corresponding geometric derivation of optimized geometry and streamline pattern on the sidewall surface is compared with those of the baseline geometry in Fig. 23. The areas of the largest derivation (colored red) seem align themselves with the shock angle, and consequently flow is conditioned by redistributing streamlines especially following the glancing shock region as well as near the wedge region. Thus, the dispersion of streamlines through reshaping the sidewall geometry reduces the glancing sidewall SWBLIs and results in a weaker corner separation.

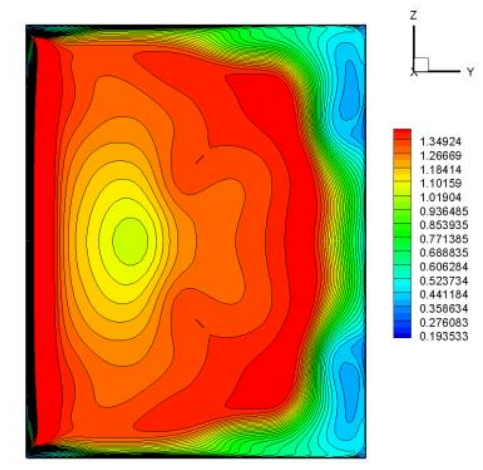

(a) Baseline (B0000)

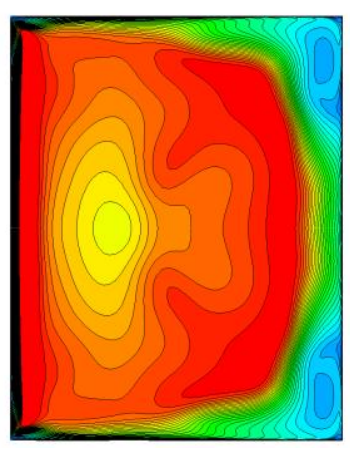

(b) $s 6011$

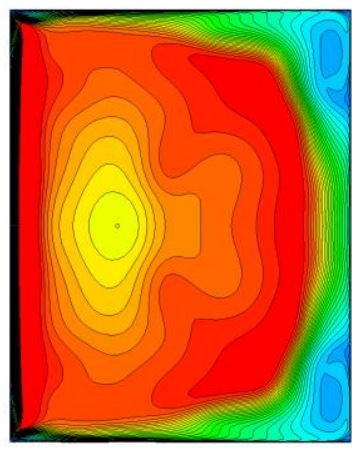

(c) s6018

Fig. 21: Pitot pressure comparison at AIP $(x=55 \mathrm{~cm})$ : (a) baseline (B0000), (b) the least distortion (s6011), (c) best PR (s6018), $2^{\text {nd }}$ order spatial accuracy. 

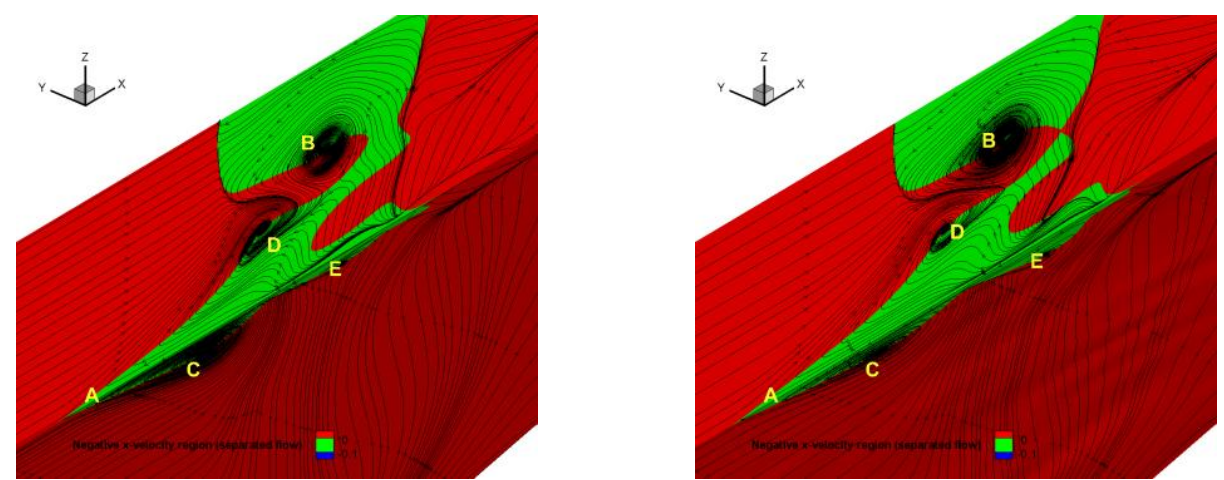

Fig. 22: Oil-flow pattern over $x$-velocity contour of baseline (B000000) and optimized geometries (s6011, best DPCP). The non-red colored regions are areas of negative axial velocity. The one on the left is the baseline and the least distortion sidewall design in on the right.

The sidewall design above shows its effectiveness to mitigate the adverse effect of corner SWBLI. However, the currently investigated designs which are constrained at $10 \%$ of the incoming boundary layer thickness $(\delta=1.37 \mathrm{~cm})$ seem not big enough to change the flow pattern drastically. Thus, the geometric constraint is relaxed from $10 \%$ to $50 \%$ (i.e. $-0.5 \mathrm{~cm} \leq \Delta z \leq 0.5 \mathrm{~cm}$ ) and tested on the sidewall design. The pitot pressure contours of the best distortion performer, design S4032, in the $40^{\text {th }}$ generation are shown on the right in Fig. 24. The optimized geometry results in a total pressure recovery of 0.8488 and DPCP of 0.1904 . This resulting optimized geometry shows very different flow characteristics especially around the corner when compared with flow in the baseline and the more constrained case (s6011). Low pitot pressure region on the bottom floor is dispersed further toward the sidewall region so that the boundary layer thickness on the bottom wall is about the same but in a much healthier profile while sidewall's boundary layer thickness gets thicker. Furthermore, the swirling at the corner is getting dramatically smaller as the amplitude of the geometric perturbation on sidewall is larger. The same flow phenomena can also be observed from the oil flow pattern comparison between the baseline and the 50\% constrained design in Fig. 25. The size of corner separations between A and C are fundamentally diminished so that the swirling at the corner in AIP plane could be removed. In addition, as the corner separation is reduced, the reverse flow region between corner and central separation on the bottom floor is also diminished. By these changes, the reduction of the distortion reaches about 50\%. The thicker boundary layer on the bottom and sidewalls, however, prevented the increase of total pressure recovery against the baseline geometry.
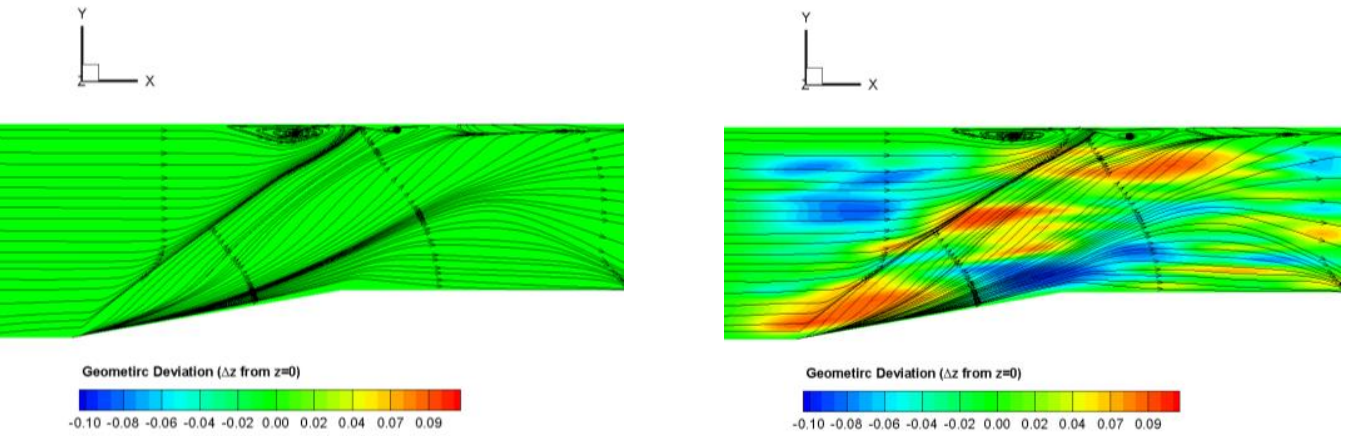

Fig. 23: Geometric deviation and oil flow pattern on the side wall: (a) baseline (left); (b) the least DPCP design (s6011, right) 


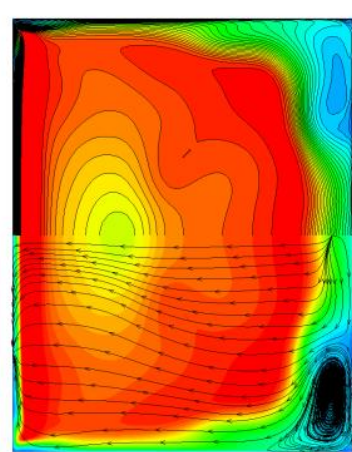

(a) Baseline (B0000)

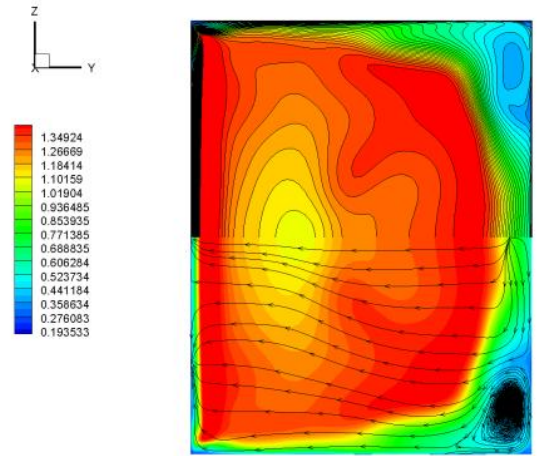

(b) DPCP best (10\%)

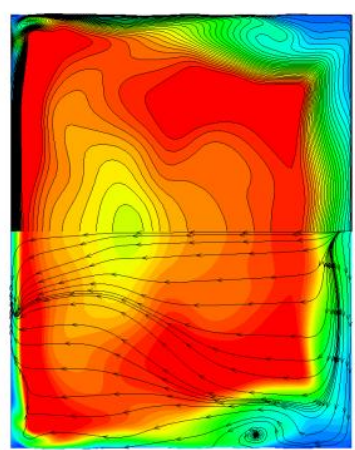

(c) DPCP best $(50 \%)$

Fig. 24: Pitot pressure and streamline comparison at AIP $(x=55 \mathrm{~cm})$ : (a) baseline (B0000); (b) the $10 \%$ constrained least distortion (s6011); (c) the 50\% constrained least distortion (S4032). All results are in $2^{\text {nd }}$ order spatial accuracy.
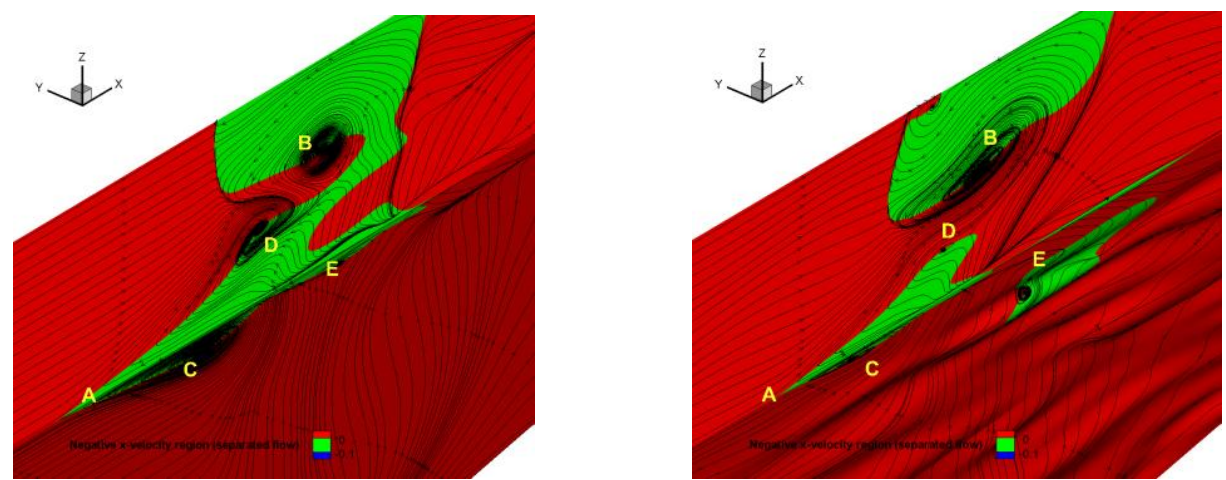

Fig. 25: Oil-flow pattern over $x$-velocity contours of the baseline (B000000) and the optimized geometry (the least DPCP) in the case of 50\% constraint with respect to BL thickness. The non-red colored regions are areas of negative axial velocity. The one on the left is the baseline and the least distortion sidewall design (S4032) is on the right. 


\section{Corner effect}

Based on the observations from bottom and side wall design problems, it can be concluded that the flow features in the 3-D SWBLIs at a rectangular supersonic inlet cannot be addressed separately because the physics behind the side, bottom, and corner interactions are highly coupled. Thus, the corner region which is connecting the walls to each other is very important to make the current design concepts more synergetic.
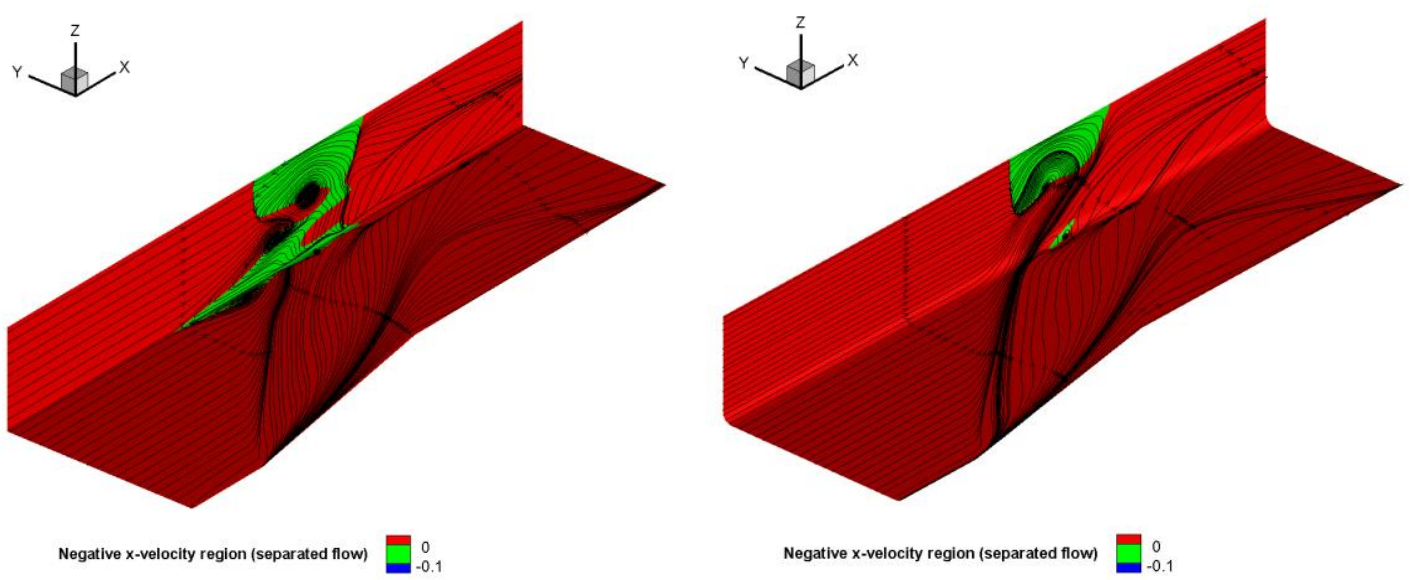

Fig. 26: Oil-flow pattern over axial ( $x$-) velocity contour . Left is the CFD results from the baseline (Oskam case) and right is from corner filleted geometry.

To address corner interaction, filleted corners approximately equivalent to the height of the incoming boundary layer are employed. Though a similar corner approach has been presented ${ }^{24}$, the current study focuses on isolating the geometry perturbations on bottom floor, side wall and corner respectively and separately. Thus, as for the corner design spaces, it is carried out a flow analysis replacing the right-angle corners of the baseline geometry with a filleted corners. In response, the flow in the filleted corner shown in the right oil pattern figure of Fig. 26 has a much smaller corner separation than the baseline. The size of central separation is similar to that of the baseline but its strength is much weakened by the smoother climb of flow stream from the side wall due to the absence of corner separation. The AIP pitot pressure contours and streamlines are compared in Fig. 27. It is observed that the filleted corner impedes the lateral migration of the subsonic portion of the sidewall boundary and results in less low energy fluid accumulated in the corner. Thereby, the size of the corner separation is significantly reduced. The total pressure recovery gain from the changing corner geometry from right-angle to the filleted one is $2.4 \%$ from 0.8604 to 0.8840 .

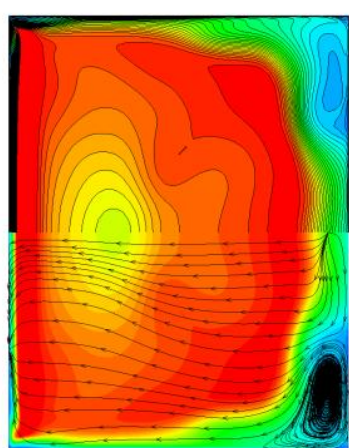

(a) Baseline (B0000)

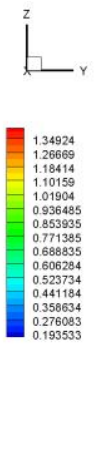

(b) Corner filleted

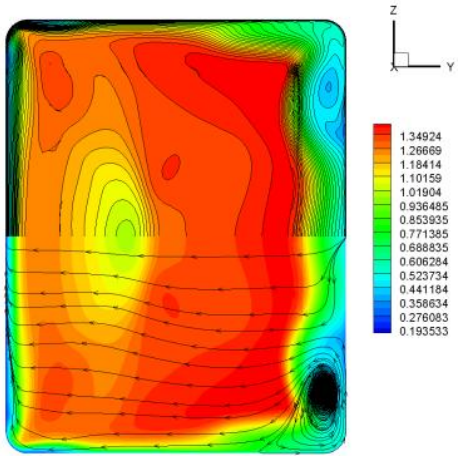

Fig. 27: Pitot pressure and streamlines comparison at AIP $(x=55 \mathrm{~cm})$ : (a) baseline (B0000), (b) Corner filleted duct, $2^{\text {nd }}$ order spatial accuracy.

Instead of performing separate optimization on each geometric component, it is believed that applying optimization upon a design space composed of bottom floor, sidewall and filleted corner (i.e. all components) will be more effective in dealing with the three-dimensional SWBLIs. However, this study of uncovering 
optimal shape on separate geometric component of a rectangular duct avails the understanding of the mechanism played by each geometric individual. Further study in shaping corner geometry has been conducted and will be presented in the future.

\section{Conclusion and Future Work}

We have considered the flow control optimization of shock wave boundary layer interactiosn governed by the three dimensional Navier-Stokes equations. In particular, we have considered objective functions motivated by minimizing flow distortion and maximizing total pressure recovery at API. Numerical shape parameterization coupled with optimization method has been conducted. Optimized shape is very effective for two-dimensional SWBLI at a single design point. More powerful optimization techniques are needed in extending operability in problem of multiple design points, such as a multi-objective optimization that incorporates all objectives at all design points into the problem. In three dimensional SWBLIs, it is found that any improvement on the bottom wall has little effect in improving total pressure though it is useful in suppressing the incident separation. Shaping side wall geometry is effective in redistributing flow on the side wall and results in a less distorted flow at the exit. A preliminary corner geometry study indicates a significant performance improvement. To further investigating the complexity of the corner interactions resulting from an oblique SWBLI and the searching for optimal corner geometry, a free-form deformation method ${ }^{25}$ is employed to represent volume deformation around interior corners of supersonic inlets and to carry out the corner design optimization.

\section{Acknowledgments}

May-Fun Liou is grateful for the support of the NASA's High Speed Project of the Fundamental Aerodynamics Program.

\section{References}

[1] Titchener, N., and Babinsky, H., "Shock Wave/Boundary Layer Interaction control Using a Combination of Vortex Generators and Bleed," AIAA Journal, Vol 51, No. 5, May 2013, pp.1221-1233.

[2] M.K. Fukuda, W.R. Hingst, and E. Reshotko, "Control of Shock Wave-Boundary Layer Interactions by Bleed in Supersonic Mixed Compression Inlets", NASA CR-2595, 1975.

[3] M.J. Rimlinger, T.I-P. Shih, and W.J. Chyu, "Shock-Wave/Boundary Layer Interactions with Bleed Through Rows of Holes," AIAA J. Propulsion and Power, 12:217-224, 1996. 2012.

Davis, D. O., Vyas, M., and Slater, J. W., "Research on Supersonic Bleed," AIAA Paper 2012-0272,

B. Anderson and J. Gibb, "Vortex-Generator Installation Studies on Steady-State and Dynamic Distortion," J. Aircr., 35: 513-520.

[6] Dudek, J. C., "Modeling Vortex Generators in a Navier-Stokes Code,", AIAA Journal, Vol 49, No. 4, April 2011, pp. 748.

[7] C.W.P. Ford and H. Babinsky, "Micro-Ramp Control for Oblique Shock Wave/Boundary Layer Interactions," AIAA-2007-4115, June 2007.

[8] Hirt, S. M., and Vyas, M. A., "Effects of Hybrid Flow Control on a Normal Shock Boundary-Layer Interaction," AIAA Paper 2013-0014.

Gunzburger, M., Perspectives in Flow Control and Optimization, SIAM, 2003.

[10] B. König, M. Pätzold, T. Lutz, and E. Krämer, "Numerical and Experimental Validation of ThreeDimensional Shock Control Bumps,” J. Aircr., 46:675-682, 2009.

[11] B.J.Lee, Meng-Sing Liou, and Chongam Kim, "Optimizing a boundary-layer-ingestion offset inlet by discrete adjoint approach,” AIAA Journal, Vol.48, No.9 (2010), pp.2008-2016.

[12] Shukla, V., Gelsey, A., Schwabacher, M., smith, D., and Knight D., "Automated Design Optimization for the P2 and P8 hypersonic Inlets," Journal of Aircraft, Vol. 34, No. 2,1997, pp. 228-235.

[13] B. H. Anderson and T. J. Benson, "Numerical Solution to the Glancing Sidewall Oblique ShockWave/Turbulent-Layer Interaction in Three Dimensions," AIAA 83-0136, Jan. 1983. 
Debb, K., Pratap, A., Agarwal, S., and Meyarivan, T, "A fast and elitist Multi-Objective Genetic Algorithum: NSGA-II," IEEE Transactions on Evolutionary Computation, Vol. 6, No. 2, pp. 181-197, 2002.

[15] Chang Sung Kim, Chongam Kim, Oh Hyun Rho, "Parallel Computations of High-Lift Airfoil Flows Using Two-Equation Turbulence Models," AIAA Journal, 2000, Vol.38: 1360-1368, 10.2514/2.1135

[16] B. Oskam, I. E. Vas, and S. M. Bogdonoff, "Oblique Shock wave/Turbulent Boundary-Layer Interactions in Three Dimensions at Mach 3," Pt. I. Air Force Flight Dynamics, Wright-Patterson Air Force Base, OH, AFFDL-TR-76-48, June 1976.

[17] D. R. Reddy and G. J. Harloff, "Three-Dimensional Viscous Flow Computations of High Area Ratio Nozzles for hypersonic Propulsion,” J. of Propulsion, Vol. 7, No. 1:84-89, 1991.

[18] K. Kapoor, B. Anderson, and R. J. Shaw, "Validation of the RPLUS3D Code for Supersonic Inlet Applications Involving Three-Dimensional Shock Wave-Boundary Layer Interactions," NASA-TM106579, Sep. 1994.

[19] Carter, M. B., Campbell, R. L., Pendergraft, O. C., Friedman, D. M., and Serrano, L., "Designing and Testing a Blended Wing Body with Boundary layer Ingestion nacelles," AIAA J. 43, pp.1479-1489.

[20] Anon., "Gas Turbine Engine Inlet Flow distortion", Society of Automotive Engineers, Report ARP1420, March 1978.

[21] Debb, K., Pratap, A., and Meyarivan, T, "Constrained Test problems for Multi-objective Evolutionary Optimization," Proceedings of the First International Conference on Evolutionary Multi-Criterion Optimization, Zurich, Switzerland, pp. 284-298, March, 2001.

[22] Reda, D. C. and Murphy, J. D., "Sidewall Boundary-Layer Influence on Shock Wave/Turbulent Boundary-Layer Interactions," AIAA J 11, No 10, 1973, pp1367-1368.

[23] Babinsky, H. "Corner effects in reflecting Oblique SBLIs ," the $6^{\text {th }}$ SWBLI Workshop, April, 2013

[24] Baruzzini, D., Domel, N., and Miller, D. N., "Addressing Corner Interactions Generated by Oblique Shock-Waves In Unswept Right-Angle Corners and Implications for High Speed Inlets,", AIAA Paper 2012-0275, 2012.

[25] Samareh, J.,“A Novel Shape Parameterization Approach,” NASA TM-1999-209116, May 1999. 